\title{
Educación emocional para el desarrollo de competencias emocionales en niños y adolescentes
}

\section{Núria Pérez Escoda}

Doctora en Ciencias de la Educación Universidad de Barcelona- España nperezescoda@ub.edu

https://orcid.org/0000-0001-6314-2792

\section{Gemma Filella Guiu}

Doctora en Ciencias de la Educación Universidad de Lleida - España.

gfilella@pip.udl.cat

https://orcid.org/0000-0002-0696-296X

Artículo de reflexión

Recepción: 7 de febrero de 2019 Aprobación: 3 de junio de 2019 https://doi.org/10.19053/22160159.v10.n25.2019.8941

\section{Resumen}

A partir de la convicción de que la educación debe preparar para la vida, en los últimos años se ha generado un movimiento a favor de promover, difundir y desarrollar la educación emocional como innovación psicopedagógica. Con un enfoque de ciclo vital, esta forma parte del currículo académico en todas sus etapas. En este artículo se aportan orientaciones para su puesta en práctica y elaborar programas en los centros educativos de cualquier nivel. Se hace una propuesta de los objetivos y contenidos curriculares más apropiados para trabajar con personas de 3 a 16 años. También se reflexiona acerca de las competencias a desarrollar, las metodologías o estrategias didácticas apropiadas y acerca de los criterios a tener en cuenta en la elaboración de los programas de educación emocional.

Palabras clave: inteligencia emocional, innovación psicopedagógica, currículo 


\title{
Emotional learning for developing emotional skills in children and adolescents
}

\begin{abstract}
On the basis of the conviction that education must prepare people for life, during the last years a movement for promotion, dissemination, and development of emotional learning as a psychopedagogical innovation has been generated. Its starting point is curricula in all stages with a life cycle approach. This article provides guidelines for its implementation as well as for program creation in educational centers at any level. A proposal is made regarding the most appropriate curricular content and objectives for the 3-16 age group. The article also reflects on the skills to be developed, the appropriate methodologies or didactical strategies, and the criteria to take into consideration for the creation of emotional learning programs.
\end{abstract}

Keywords: emotional intelligence, psycho-pedagogical innovation, curriculum

\section{Enseignement émotionnel pour le développement de compétences émotionnelles chez des enfants et des adolescents}

\section{Résumé}

Partant de la conviction que l'enseignement prépare les personnes à la vie, ces dernières années, un mouvement qui promeut, dissémine, et développe l'enseignement émotionnel en tant qu'innovation pédagogique a été généré. Son point de départ est les programmes académiques à toutes leurs étapes avec une approche de cycle de vie. Cet article contient des lignes directrices pour sa mise en œuvre ainsi que pour l'élaboration de programmes 
dans les établissements éducatifs de tous les niveaux. On propose les objectifs et les contenus des programmes scolaires les plus appropriés pour le groupe d'âge de 3 à 16 ans. On réfléchit aussi aux compétences à développer, aux méthodologies ou aux stratégies didactiques appropriées, et aux critères à prendre en compte dans l'élaboration de programmes d'enseignement émotionnel.

Mots-clés : intelligenceémotionnelle, innovation pédagogique, programme scolaire

\section{Educação emocional para o desenvolvimento de competências emocionais em crianças e adolescentes}

\section{Resumo}

A partir da convicção de que a educação deve preparar para a vida, nos últimos anos tem-se gerado um movimento em prol de promover, difundir e desenvolver a educação emocional como inovação psicopedagógica. Com um enfoque de ciclo vital, esta forma parte do currículo académico em todas suas etapas. Neste artigo contribuem-se orientações para sua posta em prática e elaborar programas nos centros educativos de qualquer nível. Faz-se uma proposta dos objetivos e conteúdos curriculares mais apropriados para trabalhar com pessoas desde os três até os dezesseis anos. Também se reflexiona a respeito das competências a desenvolver, as metodologias ou estratégias didáticas apropriadas e a respeito dos critérios a ter em conta na elaboração dos programas de educação emocional.

Palavras-chave: inteligência emocional, inovação psicopedagógica, currículo 


\section{Introducción}

Las afirmaciones que relacionaban el grado de inteligencia de una persona con la capacidad para conseguir éxito o incidir decisivamente en su vida de forma adecuada han experimentado en las últimas décadas una importante matización que supera el tópico que concebía la inteligencia como una capacidad general unitaria. Actualmente, se puede hablar de una modularidad de la inteligencia, la cual permite entender con mucha mayor facilidad que las personas difieran en el grado de acierto con el que afrontan las diversas tareas o retos cotidianos. Trabajos como los de Salovey y Mayer (1990), Gardner $(1983,1993)$ y Sternberg (1990, 2000) son representativos de los avances en la reformulación del concepto de inteligencia. En ellos se observa cómo el constructo de inteligencia ha experimentado una ampliación conceptual muy importante, especialmente a partir de la teoría de las inteligencias múltiples de Gardner (1993), la cual ha tenido una gran repercusión en la atención a la diversidad en el contexto educativo. Dicha teoría actualmente distingue nueve inteligencias, de entre las que destacamos la inteligencia interpersonal y la intrapersonal, que confieren al concepto de inteligencia una mayor proximidad a la dimensión personal y emocional.

La inteligencia intrapersonal se relaciona con la capacidad de formarse un modelo preciso de sí mismoy utilizarlo apropiadamente, el cual es preeminente en el momento de tomar decisiones esenciales a lo largo de la vida. La inteligencia intrapersonal puede equipararse al concepto de inteligencia personal de Sternberg (2000). Paralelamente, la inteligencia interpersonal -también denominada competencia social por autores como Rose-Krasnor (1997), Cherniss (2000), Topping, Bremmer y Holmes (2000) y Zirkel, (2000), entre otros- se concibe como la capacidad de comprender a los demás y relacionarse con ellos de forma mutuamente beneficiosa.

Influenciados por los trabajos sobre las inteligencias múltiples, Salovey y Mayer (1990) desarrollaron el concepto de inteligencia emocional, que definían entonces como la habilidad para manejar los sentimientos y emociones, discriminar entre ellos y utilizar esta información para distinguir entre los propios pensamientos y acciones. Esta primera acepción ha ido experimentando diversas reformulaciones (Mayer, Caruso \& Salovey, 1999, 2001; Mayer \& 
Salovey, 1993, 1997, 2007; Mayer, Salovey \& Caruso, 2000) hasta concebirse como la habilidad para percibir, valorar y expresar emociones con exactitud, acceder y generar sentimientos que faciliten el pensamiento, comprender emociones y regularlas para permitir el crecimiento emocional e intelectual (Mayer \& Salovey, 1997).

Tras la difusión de este concepto, y especialmente tras la publicación del best-seller La inteligencia emocional, de Goleman en 1995, numerosos investigadores, como Cooper y Sawaf (1997), Weisinger (1998), Dulewicz y Higg (1999), Bar-On y Parker (2000), Goleman (1999), McCrae (2000), Petrides y Furnham (2001) y Mayer, Salovey, Caruso y Sitarenios (2003), se han preocupado de este constructo y han elaborado diferentes acepciones del mismo. Así, bajo la denominación de inteligencia emocional se encuentran diversas concepciones que, tal como señalan Mayer et al. (2000), permiten entender la inteligencia emocional como mínimo de tres formas: como movimiento cultural, como rasgo de personalidad y como capacidad mental.

El debate sobre lo que es realmente inteligencia emocional está servido. Sin embargo, tal como se ha señalado en otros trabajos, la misma existencia del constructo está en discusión (Bisquerra, Pérez-González \& García, 2015). La deliberación científica acerca de este concepto es larga e interesante -en Mestre, Comunian y Comunian (2007) puede profundizarse sobre los detalles de la misma-. Curiosamente, en medio de la polémica, destaca un punto de acuerdo: la existencia de un conjunto de competencias emocionales, con un elevado valor para la vida, que pueden ser aprendidas (Bar-On \& Parker, 2000; Cohen, 1999; Elias, Tobias \& Friedlander, 1999, 2001; Elias et al., 1997; Goleman, 1995, 1999; Saarni, 2000; Salovey \& Sluyter, 1997). La implicación educativa de esta afirmación es muy clara: es preciso centrar los esfuerzos en el desarrollo de competencias emocionales y delegar a la psicología la dilucidación en torno al constructo de inteligencia emocional (Bisquerra \& Pérez-Escoda, 2007).

Este cambio de enfoque se evidencia también en una nueva terminología, pues, desde el punto de vista educativo, se prefiere hablar de educación emocional y se pone el énfasis en la interacción entre la persona y el ambiente y, como consecuencia, se confiere gran importancia al aprendizaje y progreso. La educación emocional tiene como objetivo contribuir a este desarrollo. 


\section{La educación emocional}

$\mathrm{Si}$ bien es cierto que la educación en el siglo XXI se ha generalizado hasta alcanzar a la totalidad de la población en los países desarrollados, también hay que reconocer que se ha centrado fundamentalmente en una instrucción cognitiva y olvida el desarrollo de las competencias múltiples y especialmente de las competencias emocionales.

La reflexión acerca de cómo se desarrolla la educación de niños y jóvenes ha facilitado que muchos educadores denunciaran que centrar el aprendizaje únicamente en las materias académicas ordinarias no permite atender suficientemente el desarrollo integral de las futuras generaciones.

Así, en el informe Replantear la educación (Unesco, 2015) se certifica la necesidad de superar el aprendizaje académico tradicional y de propiciar un planteamiento holístico de la educación y del aprendizaje, para superar las dicotomías tradicionales entre los aspectos cognitivos, emocionales y éticos.

Desde la convicción de que la educación debe preparar para la vida, se ha generado en los últimos años un movimiento a favor de la importancia de promover, difundir y desarrollar la educación emocional como innovación psicopedagógica. Así, se pretende dar respuesta a algunas de las necesidades socioeducativas que no quedaban hasta el momento suficientemente atendidas.

Es innegable que en los últimos años, caracterizados por una fuerte crisis, el escenario ha sido desalentador: desempleo, dificultades económicas, aumento de la pobreza, división política y corrupción, entre otros asuntos negativos con los que cada uno se despierta y sigue siendo bombardeado mediáticamente durante todo el día. No es de extrañar que algunos estudios (American Psychological Association, 2007) destaquen un aumento importante de los índices de estrés percibido en los últimos años y que el consumo de ansiolíticos y antidepresivos no haya cesado de aumentar a partir de mediados del siglo XX. Enmarcados en este contexto pesimista, ante el cual es realmente difícil no sucumbir al desánimo e impotencia, urge más que nunca defender la importancia de fortalecer la estabilidad emocional de nuestros niños y jóvenes, quienes también están creciendo inmersos en este ambiente de presión. De acuerdo con Clouder (2011), las personas 
más resilientes tienen mayores probabilidades de enfrentarse a la adversidad de modo menos dañino para su salud psicológica y física. La educación emocional fomenta la resiliencia personal (Sandoval \& López, 2017).

Aprender a motivarse, a afrontar la frustración, a controlar la ira y la conducta impulsiva, a desarrollar y difundir el sentido del humor, a generar y autogenerarse emociones positivas, a fomentar la empatía y a demorar la gratificación constituye solo una breve lista de competencias emocionales cuyo dominio permite estar mejor preparados para la vida. Entre los aspectos que se ven favorecidos por el desarrollo de las competencias emocionales están los procesos de aprendizaje, las relaciones interpersonales, la resolución de problemas y la consecución y mantenimiento de un puesto de trabajo.

La educación emocional propugna la prevención, al apostar por el empoderamiento personal de las generaciones del futuro. El desafío no es sencillo: la adquisición y utilización de competencias apropiadas en el momento oportuno no es comparable con el aprendizaje de conocimientos, conceptos o procedimientos, sino que implica una complejidad mucho mayor. Como señaló Gagné (1965), la adquisición de habilidades y competencias requiere de mucho más tiempo para alcanzar su dominio. Es preciso ofrecer múltiples oportunidades en las que poner las competencias en práctica y propiciar su uso en contextos diferentes. Además del entorno escolar, la familia y, por extensión, cualquier situación vital se convierten en escenarios oportunos para el desarrollo de competencias emocionales. Algunos autores (Bimbela, 2008; Martín, 2016; Torrabadella, 2006) proponen la creación de gimnasios emocionales diseñados como espacios donde formarse y realizar diversas actividades con la finalidad de mejorar el bienestar personal y social a través de la mejora de las competencias emocionales con el apoyo de facilitadores.

No obstante, volviendo al contexto escolar, el desarrollo de las competencias emocionales está ausente en los sistemas educativos de la mayoría de países. Los informes internacionales sobre los avances de la educación social y emocional (Fundación Botín, 2011, 2013, 2015; Fundación Marcelino Botín, 2008) recogen un conjunto de experiencias que generalmente responden a proyectos concretos, acciones ocasionales y distan de estar presentes en la 
práctica educativa de forma planificada, dispuesta y reconocida en los planes educativos institucionales.

Desde el punto de vista de la educación emocional, no es suficiente con realizar "algunas actividades" de vez en cuando. Se precisa un trabajo intencional, sistemático y efectivo que requiere de un conjunto organizado, coherente e integrado de actividades articuladas entre sí con un propósito común.

Entendemoslaeducaciónemocional,enlostérminosdeBisquerra (2009b), como un proceso educativo, continuo y permanente, que pretende potenciar el desarrollo de las competencias emocionales como elemento esencial del desarrollo integral de la persona, con objeto de capacitarla para la vida. El concepto de educación emocional es más amplio que el de inteligencia emocional. En él se recogen aportaciones de la neurociencia, de las investigaciones sobre la psicología positiva y el bienestar subjetivo, el concepto de fluir, entre otros. Aunque se fundamenta en la inteligencia emocional, posee un carácter integrador y abierto que ha incorporado -y sigue receptivo a aceptar- nuevos avances.

La educación emocional adopta un enfoque de ciclo vital, por lo que es preciso que forme parte del currículo académico a lo largo de todas las etapas educativas, desde la educación infantil hasta la edad adulta y se extienda a la formación permanente durante toda la vida. La educación emocional es una forma de prevención primaria inespecífica que pretende minimizar la vulnerabilidad de la persona a determinadas disfunciones, o prevenir su ocurrencia (Bisquerra, 2009a, 2011, 2016).

El objetivo principal de la educación emocional es el desarrollo de competencias emocionales y el bienestar (Bisquerra \& Pérez-Escoda, 2007). Este objetivo general puede concretarse en objetivos más específicos que se derivan de las diversas competencias emocionales.

\section{Competencias emocionales}

Las competencias emocionalessonel conjuntodeconocimientos, capacidades, habilidades y actitudes necesarias para comprender, expresar y regular de forma apropiada los fenómenos emocionales.

La delimitación de las competencias emocionales aparece como un tema de debate en el que no existe un acuerdo unánime entre los 
expertos. En Bisquerra y Pérez-Escoda (2007) puede encontrarse una revisión de las principales propuestas de clasificación de las competencias emocionales a partir de la cual se establece un modelo propio (figura 1), que las agrupa en cinco bloques: conciencia emocional, regulación emocional, autonomía personal, competencia social y competencias para la vida y el bienestar.

El primero de los bloques, conciencia emocional, alude a la capacidad para tomar conciencia de las propias emociones y de las emociones de los demás, incluyendo la habilidad para captar el clima emocional de un contexto determinado.

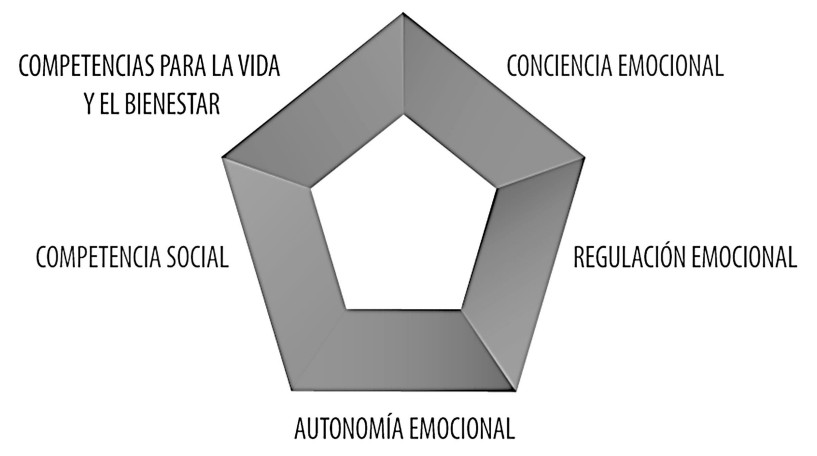

Figura 1: Modelo pentagonal de competencias emocionales. Adaptado de Bisquerra y Pérez-Escoda (2007, p.70).

La regulación emocional, por su parte, designa la capacidad para manejar las emociones de forma apropiada. Supone tomar conciencia dela relación entre emoción, cognición y comportamiento y tener buenas estrategias de afrontamiento, capacidad para autogenerarse emociones positivas, entre otras.

La autonomía emocional se puede entender como un concepto amplio que incluye un conjunto de características y elementos relacionados con la autogestión personal, entre las que se encuentran la autoestima, actitud positiva antela vida, responsabilidad, capacidad para analizar críticamente las normas sociales, la capacidad para buscar ayuda y recursos, así como la autoeficacia emocional.

La competencia social es la capacidad para mantener buenas relaciones con otras personas. Esto implica dominar las habilidades sociales, capacidad para la comunicación efectiva, respeto, actitudes prosociales, asertividad, entre otras. 
Finalmente, el bloque de competencias para la vida y el bienestar incluye la capacidad para adoptar comportamientos apropiados y responsables para afrontar satisfactoriamente los desafíos diarios de la vida, ya sean privados, profesionales o sociales, sin olvidar las situaciones excepcionales con las que cada cual va tropezando. Permiten organizar la vida de forma sana y equilibrada, al facilitar experiencias de satisfacción o bienestar.

\section{Contenidos de la educación emocional para niños y adolescentes}

A partir de este marco teórico, se propone un conjunto de objetivos y contenidos para el desarrollo de programas de educación emocional en las distintas etapas educativas, que surgen como consecuencia de contemplar los siguientes criterios:

- Atender a las necesidades de desarrollo emocional en cada una de las etapas evolutivas.

- Tener en cuenta la legislación educativa en relación con las competencias a desarrollar en cada nivel y curso.

- Desarrollar actividades de aprendizaje que puedan ser aplicables a todo el grupo de clase, sin olvidar la necesaria atención a la diversidad.

- Orientarse al desarrollo de la reflexión y toma de conciencia de las emociones propias y de los demás.

- Enfocarse en la práctica vivencial para fomentar el desarrollo de competencias.

- Diseñar intervenciones con cierto margen de flexibilidad, de forma que, lejos de constituir un recetario a cumplir de forma taxativa, permita adaptar el programa en función de lo que vaya sucediendo durante la aplicación.

- Evaluar permanentemente los progresos y los procesos para poder mejorar de forma continua y progresiva los cambios necesarios en el programa.

Tener en cuenta estos criterios implica aceptar como imprescindible que los programas se adapten en todo momento a los destinatarios según sus circunstancias concretas: experiencia, conocimientos previos y nivel de madurez. Cualquier intervención 
debe partir de una evaluación de necesidades que permita analizar el nivel de desarrollo emocional previo de los destinatarios. Con ello se ajustarían mejor los objetivos y contenidos prioritarios a atender, para contextualizar la intervención. En este proceso pueden utilizarse cuestionarios, rejillas de observación y grupos de discusión. Resulta imprescindible la colaboración del profesorado que se quiera implicar en el programa.

En este sentido, conviene señalar que otro elemento clave, previo a la implantación de programas de educación emocional, es la formación de los adultos o educadores encargados de su aplicación. La educación emocional requiere que el adulto actúe como referente al que los educandos puedan imitar. Su forma de reaccionar es observada por los niños y su estado emocional se transmite y se contagia. La comunicación tanto verbal como no verbal adquiere una importancia capital. Los gestos, el tono de voz, la capacidad de escucha, el contacto físico, la regulación del enfado, la empatía, la gestión de un conflicto en el aula, el sentido del humor, la actitud comprensiva, entre otros, no pasan en absoluto desapercibidos a los ojos de los niños y adolescentes. El profesor, con sus actitudes y comportamientos, tiene las claves para propiciar un clima de seguridad, confianza, respeto, cordialidad y afecto con sus alumnos. En considerables ocasiones se ha destacado que todo proceso de educación emocional en el contexto escolar debe empezar por la formación del profesorado (Bisquerra, 2005; Bisquerra \& García, 2018; Bisquerra \& Pérez-Escoda, 2009; López-Goñi \& Goñi, 2012; Pérez-Escoda, Berlanga \& Alegre, 2019; Torrijos, Hernández \& Rodríguez, 2016).

Los contenidos de la educación emocional, a partir del modelo pentagonal de las competencias emocionales, incluyen multitud de temas entre los que destacamos los siguientes: concepto de emoción y tipos de emociones - positivas y negativas, básicas, derivadas, ambiguas, estéticas-; características de las emociones principales -miedo, ira, ansiedad, tristeza, vergüenza, aversión, alegría, amor, humor, felicidad...-; los fenómenos afectivos emoción, sentimiento, afecto, estado de ánimo...-; emoción y salud; emoción y motivación; autoconocimiento emocional y reconocimiento de las emociones de los demás; la tolerancia a la frustración, el manejo de la ira, la capacidad para retrasar gratificaciones y estrategias de regulación emocional -relajación, 
respiración, distracción conductual, reestructuración cognitiva, visualización positiva, diálogo interno...-; características de las emociones - causas, predisposición a la acción, contagio emocional...-; comportamientos socioemocionales -escucha, empatía, asertividad, técnicas de resolución de conflictos, prosocialidad...-; actitud positiva ante la vida, responsabilidad ética y social, análisis crítico de las normas sociales y las emociones en la toma de decisiones; y fluir, automotivación, bienestar subjetivo y calidad de vida.

La dimensión ética y moral también debe estar presente en el desarrollo de los contenidos de la educación emocional. Disponer de buenas competencias emocionales no garantiza que sean utilizadas con propósitos afines al logro del bienestar personal y colectivo, por lo que es preciso prevenir una aplicación deshonesta de las mismas. Por este motivo se requiere que los contenidos emocionales vayan acompañados de principios éticos (Bisquerra, 2009a, 2011; Marina, 2005).

La elección de estos contenidos y el nivel de profundización con el que se trate cada uno de ellos se ajustará a las necesidades de los destinatarios. En este sentido es muy importante tomar en consideración el proceso de desarrollo emocional de los niños y adolescentes, así como las innovadoras aportaciones de las investigaciones neurocientíficas aplicadas a la educación.

A partir de los trabajos de Bisquerra (2009a, 2016), Garaigordobil (2018), Illinois State Board of Education (2014), Ribes, Bisquerra, Agulló, Filella y Soldevila (2005), Soldevila, Filella, Ribes y Agulló (2007) y López (2011), se presenta en la tabla 1 una propuesta de secuenciación de los contenidos de la educación emocional a lo largo del currículo. 
Tabla 1

Propuesta de secuenciación de los contenidos de la educación emocional a lo largo del currículo

\section{Propuesta de secuenciación de los contenidos de la educación emocional}

Reconocimiento de las emociones de tristeza, miedo, rabia, alegría, amor-estima, humor y felicidad, a partir de la comunicación verbal y no verbal en uno mismo y en los demás.

Expresión de las emociones a nivel verbal y no verbal en situaciones reales o simuladas.

Comprender las reacciones comportamentales que nos provocan las emociones.

\section{Educación \\ Infantil (3-6 años)}

Reconocimiento de las emociones estéticas.

Aprendizaje de estrategias de respiración y relajación como estrategia de regulación (con ayuda).

La expresión emocional como estrategia de regulación (con ayuda).

La distracción conductual como estrategia de regulación (con ayuda).

Imaginación proyectiva mediante el juego simbólico.

Reconocimiento de la propia valía personal y capacidad de cierta actuación autónoma (con ayuda).

Respeto y valoración de los demás.

Ayuda al otro (dirigida y espontánea).

Continuación y profundización de todos los contenidos trabajados en la etapa anterior.

Práctica de la identificación consciente de las emociones experimentadas en diferentes momentos y situaciones.

Reconocimiento y expresión de las emociones orgullo, vergüenza, sorpresa, celos, ansiedad, a partir de la comunicación verbal y no verbal en uno mismo y en los demás.

Toma de conciencia de la subjetividad de las emociones en uno mismo.

Educación

Primaria

Aprendizaje de estrategias de reencuadre y planificación en la

(6-8 años) resolución de problemas como estrategia de regulación (con ayuda).

Aceptación de la propia responsabilidad en la regulación de la conducta (con ayuda).

Práctica de técnicas para el control de la impulsividad (demora de la gratificación).

El diálogo en la resolución de conflictos.

La cooperación y la ayuda entre compañeros.

Compartir experiencias y objetos (con ayuda).

El reconocimiento de las propias virtudes y las de los demás.

Sentimiento de pertenencia (mi grupo, mi familia, mis amigos). 


\begin{tabular}{|c|c|}
\hline \multirow{13}{*}{$\begin{array}{c}\text { Educación } \\
\text { Primaria } \\
\text { (8-10 años) }\end{array}$} & $\begin{array}{l}\text { Continuación y profundización de todos los contenidos trabajados en } \\
\text { las etapas anteriores. }\end{array}$ \\
\hline & $\begin{array}{l}\text { Identificación de dos emociones de la misma familia ante una } \\
\text { situación. }\end{array}$ \\
\hline & Clasificación de las emociones en positivas y negativas. \\
\hline & Ampliación del vocabulario emocional. \\
\hline & Distinción entre miedo y angustia. \\
\hline & $\begin{array}{l}\text { Expresión de las emociones a través de la música, el dibujo, la } \\
\text { plástica, la danza... }\end{array}$ \\
\hline & $\begin{array}{l}\text { Reconocimiento de las diferentes reacciones emocionales frente a } \\
\text { una situación determinada. }\end{array}$ \\
\hline & $\begin{array}{l}\text { Conocimiento y práctica de diferentes técnicas de relajación: } \\
\text { respiración, masaje, tensión-relajación muscular (con ayuda). }\end{array}$ \\
\hline & $\begin{array}{l}\text { Uso de la relajación conductual y cognitiva como técnica de } \\
\text { regulación (con ayuda). }\end{array}$ \\
\hline & Reconocimiento de las propias cualidades y limitaciones. \\
\hline & Conducta cooperativa. \\
\hline & Regular la frustración (con ayuda). \\
\hline & $\begin{array}{l}\text { Comprensión emocional de la conducta de los demás a partir de } \\
\text { características de personalidad. }\end{array}$ \\
\hline \multirow{12}{*}{$\begin{array}{c}\text { Educación } \\
\text { Primaria } \\
\text { (10-12 años) }\end{array}$} & $\begin{array}{l}\text { Continuación y profundización de todos los contenidos trabajados en } \\
\text { las etapas anteriores. }\end{array}$ \\
\hline & $\begin{array}{l}\text { Ampliación y precisión en el uso del vocabulario emocional: las } \\
\text { familias de emociones. }\end{array}$ \\
\hline & $\begin{array}{l}\text { Aprendizaje de nuevas emociones: compasión, sorpresa, esperanza, } \\
\text { envidia. }\end{array}$ \\
\hline & $\begin{array}{l}\text { Comprensión de la ambivalencia emocional (experiencia simultánea } \\
\text { de emociones contradictorias). }\end{array}$ \\
\hline & $\begin{array}{l}\text { Resolución autónoma de los conflictos y retos relacionales. Implica la } \\
\text { aplicación espontánea de las competencias adquiridas. }\end{array}$ \\
\hline & $\begin{array}{l}\text { Uso de la relajación conductual y cognitiva como técnica de } \\
\text { regulación (autónomamente) }\end{array}$ \\
\hline & Desarrollo progresivo de la empatía y de las actitudes prosociales. \\
\hline & $\begin{array}{l}\text { Identificación de los componentes de la respuesta emocional } \\
\text { (cognitivo, fisiológico y conductual). }\end{array}$ \\
\hline & Las relaciones de amistad, la confianza y el apoyo mutuo. \\
\hline & $\begin{array}{l}\text { Ejercicios y actividades relacionados con la toma de decisiones y } \\
\text { aceptación de responsabilidades. }\end{array}$ \\
\hline & El respeto y la transgresión de las normas sociales. \\
\hline & La organización del tiempo. \\
\hline
\end{tabular}




\begin{tabular}{cl}
\hline & Continuación y profundización de todos los contenidos trabajados en \\
& las etapas anteriores. \\
& Conciencia de los estados afectivos y las consecuencias de los \\
mismos. & Comprensión de múltiples factores contextuales para explicar las \\
& reacciones de los demás. \\
& Comprensión de las preferencias, la personalidad y la historia previa \\
& en la comprensión de las emociones de los demás. \\
& Ejercicios y actividades de educación emocional diversos: asertividad, \\
ESO & resistencia a la presión de grupo, control del impulso, tolerancia a la \\
frustración, aprender a tomar decisiones, saber pedir ayuda... \\
Aprender a automatizarse a partir de la emoción. Positivización de las \\
situaciones. \\
Búsqueda del autoconocimiento y necesidad de la autoestima. \\
Modulación del estado de ánimo. \\
Educación emocional y desarrollo moral: desarrollo del juicio crítico, \\
autocrítica para mejorar, aceptación de puntos de vista distintos, \\
respeto a la diversidad... \\
Adquisición y práctica de hábitos saludables.
\end{tabular}

Fuente: Elaboración propia.

\section{Metodología de implementación de la educación emocional}

La metodología educativa más eficaz para el desarrollo de las competencias emocionales es aquella que toma en consideración los conocimientos previos de los niños y adolescentes y tiene en cuenta sus vivencias, intereses y necesidades personales y sociales.

La práctica de la educación emocional debe llevarse a cabo fundamentalmente mediante metodologías vivenciales y participativas que contemplen actividades diversas y favorezcan la introspección y el autoanálisis: role-playing, grupos de discusión, dramatización... El adulto debe legitimar las emociones de los participantes, permitir su expresión y propiciar un clima de confianza donde se puedan regular adecuadamente las emociones desagradables, así como potenciar las emociones positivas.

Es importante aprovechar las situaciones que se generan habitualmente de forma natural y espontánea en la clase, en el recreo, o en cualquier momento de la cotidianidad, y analizarlas para favorecer la comprensión emocional y el aprendizaje funcional de la educación emocional. Se tendrá en consideración la teoría del 
aprendizaje social de Bandura (1987), al destacar la importancia de los modelos en el proceso de aprendizaje y poner especial atención en que lo que el adulto muestra con su conducta ejerce un impacto mayor que lo que dice, tanto en las actitudes como en las creencias, valores y comportamientos del alumnado.

Según las edades se elegirá en cada momento la utilización de los recursos didácticos y dinámicas más apropiados: imágenes, fotografías, la música y las canciones, cuentos, juegos, vídeos, objetos familiares o extraños, títeres, noticias de prensa, razón dialógica, imaginación emocional, dibujos, casos simulados, libros, actividades de relajación... La realización de actividades variadas que permitan alternar momentos de reflexión personal con espacios de trabajo en grupo, en las que compartir, expresar y contrastar los puntos de vista, son excelentes para favorecer el desarrollo emocional (Bisquerra et al. 2009). El uso de la música moviliza enormemente la vivencia emocional y permite experimentar ocasiones para fluir a partir de los distintos ritmos y estilos, los mensajes de las canciones y las asociaciones atribuidas a las mismas. Los objetos, los títeres, los personajes de los vídeos o cuentos permiten trabajar la perspectiva emocional de los demás, analizar otras realidades y expresar o proyectar a través de ellos las propias preocupaciones e inquietudes. Las actividades lúdicas -juegos, role-playing, discusión grupal y otras- permiten la interacción y la expresión libre de sentimientos y emociones o la escenificación empática de personajes que facilitan el crecimiento emocional. Como principio básico se respetará en todo momento la libertad de expresar o reservar sus emociones, opiniones o vivencias personales. Nunca el alumno ha de sentirse violentado, por lo que el dinamizador tendrá especial cuidado y sensibilidad al proponer los ejercicios a realizar. En este punto, Bisquerra (2009a) sugiere tratar el caso de terceras personas o, si lo considera oportuno, presentar situaciones o vivencias personales del educador con el propósito de generar un clima de confianza mutua que invite a la generosidad de compartir voluntariamente otras experiencias personales de los alumnos participantes.

El camino está iniciado. Las experiencias afortunadamente se multiplican; se observa un creciente interés por el tema. Todo esfuerzo a favor de la educación emocional merece la pena, pues tenemos como propósito la mejora del bienestar personal y social. 


\section{Conclusión}

Es necesario aportar orientaciones que faciliten el diseño y aplicación de propuestas de educación emocional ante la evidencia aportada por la investigación científica de la importante función que la mejora de las competencias emocionales ejerce sobre el alumnado en la predisposición al aprendizaje, en la reducción de la conflictividad, reducción de comportamientos de riesgo, mejora del clima del aula, entre otros beneficios.

La educación emocional pone el énfasis en la interacción entre la persona y el ambiente, y como consecuencia, se confiere gran importancia al aprendizaje y al progreso. Aprender a motivarse, a afrontar la frustración, a controlar la ira, a desarrollar y difundir el sentido del humor y a fomentar la empatía constituye solo una breve lista de competencias emocionales cuyo dominio permite estar mejor preparados para la vida. Tener en cuenta este proceso de aprendizaje conlleva en el marco educativo diseñar intervenciones adaptadas a los destinatarios con una metodología activa y motivadora.

Corresponde a los responsables educativos de cada país establecer los estándares educativos, sin embargo, en su ausencia, cada centro educativo o equipo de profesores que desean, desde la innovación educativa, implementar la educación emocional de forma coherente y secuenciada necesitan de algunas orientaciones para el diseño y puesta en práctica de sus programas.

Por ello se presenta en este trabajo una propuesta curricular, desde un enfoque del ciclo vital, que intenta facilitar al profesorado la selección de contenidos y habilidades adecuados para desarrollar en cada una de las etapas educativas. En este sentido, conviene recordar que un elemento clave previo a la implementación de programas de educación emocional: la formación de los educadores encargados de su aplicación. 


\section{Referencias}

American Psychological Association. (2007). Stress: a major health problem in the U.S, warns APA. Recuperado de http://www.apa.org/ news/press/releases/2007/10/stress.aspx

Bandura, A. (1987). Teoría del Aprendizaje Social. Madrid: Espasa-Calpe.

Bar-On, R., \& Parker, J. (Eds.). (2000). The Handbook of Emotional Intelligence. Theory, Development, Assessment, and Application at Home, School, and in the Workplace. San Francisco, Ca: Jossey-Bass.

Bimbela, J. (2008). Gimnasia Emocional. Pasamos la acción. Sevilla: Escuela Andaluza de Salud Pública.

Bisquerra, R. (2005). La educación emocional en la formación del profesorado. Revista Interuniversitaria de Formación del profesorado, 19(3), 95-114.

Bisquerra, R. (2009a). Psicopedagogía de las emociones. Madrid: Síntesis.

Bisquerra, R. (2009b). Orientació psicopedagògica, educació emocional i ciutadania. Aloma: Revista de Psicologia, Ciències de l'Educació i de l'Esport, 23-24, 119-128.

Bisquerra, R. (2016). 1o ideas clave Educación Emocional. Barcelona: Graó.

Bisquerra, R. (Coord.). (2011). Educación Emocional. Propuestas para educadores y familias. Bilbao: Desclée de Brouwer.

Bisquerra, R., \& García, E. (2018). La educación emocional requiere formación del profesorado. Revista del Consejo Escolar del Distrito, $5(8), 13-28$.

Bisquerra, R., \& Pérez-Escoda, N. (2007). Las competencias emocionales. Educación XXI, 10, 61-82.

Bisquerra, R., \& Pérez-Escoda, N. (2009). El saber hacer emocional, destreza ineludible. Cuadernos de Pedagogía, 389, 54-56.

Bisquerra, R., Pérez-González, J., \& García, E. (2015). Inteligencia emocional en la educación. Madrid: Síntesis.

Cherniss, C. (2000). Social and emotional competence in the workplace. En R. Bar-On \& J. Parker, The handbook of emotional intelligence. Theory, development, assessment, and application at home, school, and in the workplace (pp. 433-459). San Francisco, Ca: Jossey-Bass.

Clouder, C. (2011). Una nueva mirada. En Fundación Botín, Educación emocional y social. Análisis internacional, 2011. (pp. 1-278) Santander: Fundación Botín. 
Cohen, J. (Ed.). (1999). Educating minds and hearts. Social emotional learning and the passage into adolescence (pp. 3-23). Nueva York: Teachers College, Columbia University.

Cooper, R., \& Sawaf, A. (1997). Estrategia emocional para ejecutivos. Barcelona: Martínez Roca.

Dulewicz, V., \& Higgs, M. (1999). Can emotional intelligence be measured and developed? Leadership \& Organization Development Journal, 2O, 242-252. http://dx.doi.org/10.1108/01437739910287117

Elias, M., Zins, J., Weissberg, R., Frey, K., Greenberg, M., Haynes, N... Shriver, T. (1997). Promoting social and emotional learning. Guidelines for Educators. Alexandria, Virginia: Association for Supervision and Curriculum Development.

Elias, M., Tobias, S., \& Friedlander, B. (1999). Educar con inteligencia emocional. Barcelona: Plaza Janés.

Elias, M., Tobias, S., \& Friedlander, B. (2001). Educar adolescentes con inteligencia emocional. Barcelona: Plaza Janés.

Fundación Botín. (2011). Educación emocional y social. Análisis internacional, 2011. Santander: Fundación Botín.

Fundación Botín. (2013). Educación emocional y social. Análisis internacional. Informe Fundación Botín 2013. Santander: Fundación Botín.

Fundación Botín. (2015). Educación emocional y social. Análisis internacional. Informe Fundación Botín 2015. Santander: Fundación Botín.

Fundación Marcelino Botín. (2008). Educación emocional y social. Análisis internacional. Informe Fundación Botín 2008. Santander: Fundación Marcelino Botín.

Gagné, R. (1965). The conditions of learning. New York: Holt, Rinehart, and Winston.

Garaigordobil, M. (2018). La educación emocional en la infancia y la adolescencia. Participación Educativa, 5(8), 105-128

Gardner, H. (1983). Frames of mind. The theory of multiple intelligences. Nueva York: Basic Books.

Gardner, H. (1993). Multiple intelligences: The theory in practice. Nueva York: Basic Books.

Goleman, D. (1995). Inteligencia emocional ( $15^{\mathrm{a}}$ ed.). Barcelona: Kairós (Reimpreso de Emotional intelligence: Why it can matter more than IQ. 1995. Nueva York: Bantam Books). 
Goleman, D. (1999). La práctica de la inteligencia emocional. Barcelona: Kairós.

Illinois State Board of Education. (2014). Standards for Social/Emotional Learning (SEL). Recuperado de https://casel.org/in-the-district/ standards/

López, E. (2011). Educar las emociones en la infancia (de o a 6 años): reflexiones y propuestas prácticas. Madrid: Wolters Kluwer.

López-Goñi, I., \& Goñi, J. (2012). La competencia emocional en los currículos de formación inicial de los docentes. Un estudio comparativo. Revista de Educación, 357, 205-206.

Marina, J. (2005). Precisiones sobre la educación emocional. Revista Interuniversitaria de Formación del profesorado, 3(19), 27-43.

Martín, A. (2016). Gimnasios Emocionales. En Bisquerra, R. (Coord.), Gimnasia emocional y coaching. Barcelona: Horsori Editorial S.L.

Mayer, J., \& Salovey, P. (1993). The intelligence of emotional intelligence. Intelligence, 17, 433-442.

Mayer, J., \& Salovey, P. (1997). What is emotional intelligence?. En P. Salovey \& D. Sluyter, Emotional development and emotional intelligence (pp. 3-31). Nueva York: Basic Books.

Mayer, J., \& Salovey, P. (2007). ¿Qué es inteligencia emocional?. En J. Mestre \& P. Fernández (coords.), Manual de inteligencia emocional (pp. 25-45). Madrid: Pirámide.

Mayer, J., Caruso, D., \& Salovey, P. (1999). Emotional intelligence meets traditional standards for an intelligence. Intelligence, 27, 267-298. http://dx.doi.org/10.1016/So16o-2896(99)ooo16-1

Mayer, J., Caruso, D., \& Salovey, P. (2001). Emotional intelligence meets traditional standards for an intelligence. Intelligence, 29, 6.

Mayer, J., Salovey, P., Caruso, D., \& Sitarenios, G. (2003). Measuring emotional intelligence with the MSCEIT V.2.o. Emotion, 3, 97-105.

Mayer, J., Salovey, P., \& Caruso, D. (2000): Emotional Intelligence. En R. Sternberg (2000), Handbook of Intelligence (pp. 396-421). Nueva York: Cambridge University Press.

McCrae, R. (2000). Emotional intelligence from the perspective of the fivefactor model of personality. En R. Bar-On \& J. Parker, The handbook of emotional intelligence. Theory, development, assessment, and application at home, school, and in the workplace (pp. 263-277). San Francisco, Ca: Jossey-Bass. 
Mestre, J., Comunian, A., \& Comunian, M. (2007). Inteligencia emocional: una revisión a sus primeros quince años y un acercamiento conceptual desde los procesos psicológicos. En J. Mestre \& P. Fernández (Coords.), Manual de inteligencia emocional (pp. 47-68). Madrid: Pirámide.

Pérez-Escoda, N., Berlanga, V., \& Alegre, A. (2019). Desarrollo de competencias socioemocionales en educación superior: evaluación del posgrado en educación emocional. Bordón, 71(1), 97-113. https://doi. org/10.13042/Bordon.2019.64128

Petrides, K., \& Furnham, A. (2001). Trait emotional intelligence: Psychometric investigation with reference to established trait taxonomies. European journal of personality, 15(6), 425-448.

Ribes, R., Bisquerra, R., Agulló, M., Filella, G., \& Soldevila, A. (2005). Una propuesta de currículum emocional en educación infantil (3-6 años). Cultura y educación, 17(1), 5-17.

Rose-Krasnor, L. (1997). The nature of social competence: a theoretical review. Social development, 6, 111-135. https://doi. org/10.1111/j.1467-9507.1997.tbooo97.x

Saarni, C. (2000). Emotional competence. A developmental perspective. En R. Bar-On \& J. Parker (Eds.), The handbook of emotional intelligence. Theory, development, assessment, and application at home, school, and in the workplace (pp. 68-91). San Francisco, Ca: Jossey-Bass.

Salovey, P., \& Mayer, J. (1990). Emotional Intelligence. Imagination, Cognition, and Personality, 9, 185-211.

Salovey, P., \& Sluyter, D. (Eds.). (1997). Emotional development and emotional intelligence. Educational implications. Nueva York: Basic Books.

Sandoval, C., \& López, O. (2017). Educación, psicología y coaching: un entramado positivo. Educatio Siglo XXI, 35(1), 145-164. https://doi. org $/ 10.6018 / \mathrm{j} / 286261$

Soldevila, A., Filella, G., Ribes, R., \& Agulló, M. (2007). Una propuesta de contenidos para desarrollar la conciencia y la regulación emocional en la Educación Primaria. Cultura y Educación, 19(1), 47-59.

Sternberg, R. (1990). Metaphors of mind: Conceptions of the nature of intelligence. New York: Cambridge University Press.

Sternberg, R. (2000). Handbook of Intelligence. Nueva York: Cambridge University Press.

Topping, K., Bremmer, W., \& Holmes, E. (2000). Social competence: the social construction of the concept. En R. Bar-On \& J. Parker. 
The handbook of emotional intelligence. Theory, development, assessment, and application at home, school, and in the workplace (pp. 28-39). San Francisco, Ca: Jossey-Bass.

Torrabadella, P. (2006). Gimnasia Emocional. Barcelona: Obelisco.

Torrijos, P., Hernández, J., \& Rodríguez, M. (2016). Desarrollo de competencias emocionales en los futuros docentes de Educación Secundaria: resultados de la aplicación de un programa formativo. Revista Educativa Hekademos, 2(1), 35-43.

Unesco. (2015). Replantear la educación. ¿Hacia un bien común mundial? París: Unesco.

Weisinger, A. (1998). La inteligencia emocional en el trabajo. Buenos Aires: Javier Vergara.

Zirkel, S. (2000). Social intelligence: the development and maintenance of purposive behavior. En R. Bar-On \& J. Parker. The handbook of emotional intelligence. Theory, development,assessment, and application at home, school, and in the workplace (pp. 3-27). SanFrancisco, Ca: Jossey-Bass. 